\title{
L'accès des particuliers à la justice constitutionnelle au Burundi : le juge qui censure le constituant.
}

\author{
Par Bernard Ntahiraja ${ }^{1}$
}

SIGLES ET ABREVIATIONS

$\begin{array}{ll}\text { A.R: } & \text { Arrêté royal } \\ \text { A.N: } & \text { Assemblée Nationale } \\ \text { A.N.T: } & \text { Assemblée Nationale de Transition } \\ \text { B.O.B : } & \text { Bulletin Officiel du Burundi } \\ \text { C.C : } & \text { Cour constitutionnelle } \\ \text { CNDD-FDD : } & \text { Conseil National pour la Défense de la Démocratie-Force } \\ & \text { pour la Défense de la Démocratie } \\ \text { FRODEBU : } & \text { Front pour la Démocratie au Burundi } \\ \text { Ibidem : } & \text { Même auteur, même ouvrage, même page } \\ \text { Idem : } & \text { Même auteur, même ouvrage } \\ \text { Loc.cit : } & \text { au même lieu que précédemment } \\ \text { R.C.C.B : } & \text { Rôle de la Cour constitutionnelle du Burundi } \\ \text { Op. cit : } & \text { Opere citato (ouvrage déjà cité) } \\ \text { S.T : } & \text { Sénat de transition } \\ \text { U.B.J : } & \text { Union burundaise des journalistes } \\ \text { UPRONA : } & \text { Unité et Progrès national }\end{array}$

\section{Introduction générale}

En date du 18 mars 2005, au sortir d'une longue guerre civile ayant duré plus d'une décennie, le Burundi se dote d'une nouvelle constitution. L'attachement aux principes de l'état de droit est affirmé dans les tout premiers paragraphes du préambule. ${ }^{2}$ Cet attachement est d'autant plus prononcé que le constituant y revient dans les valeurs fondamentales sur lesquelles les Burundais entendent reconstruire leur nation. ${ }^{3}$ Le constituant ne faisait d'ailleurs ainsi que suivre la ligne déjà tracée par l'Accord d'Arusha pour la paix et la réconciliation au Burundi, signé le 28 août 2000. ${ }^{4}$

1 Bernard Ntahiraja est enseignant à la Faculté de Droit de l'Université du Burundi et doctorant à la Katolieke Universiteit Leuven (KUL) en Belgique. Il peut être contacté par e-mail à l'adresse suivante: ntahibern@yahoo.fr.

2 Le troisième paragraphe du préambule dispose : "...Considérant la nécessité de réinstaurer un ordre démocratique pluraliste et un Etat de droit; » (c'est nous qui soulignons).

3 L'article 18, al.2 dispose : "Le Gouvernement respecte la séparation des pouvoirs, la primauté du droit et les principes de la bonne gouvernance et de la transparence dans la conduite des affaires publiques. » (C'est nous qui soulignons).

4 L'article $1^{\mathrm{er}}$, al. 6 de cet accord dispose : «La fonction du régime politique est d'unir, de rassurer et de réconcilier tous les Burundais, tout en veillant à ce que le Gouvernement puisse être au service 
L'un des moyens classiques d'asseoir l'état de droit est l'instauration d'une justice constitutionnelle opérationnelle. Bâtissant sur les principes déjà posés par la constitution du 13 mars1992 qui a rouvert le Burundi au pluralisme politique ${ }^{5}$, la constitution du 18 mars 2005 organise un système de justice constitutionnelle largement ouvert et très stimulant. En termes d'ouverture à la mise en œuvre, le constituant de 2005 accorde le droit de saisine de la Cour constitutionnelle, non seulement à quelques autorités de l'Exécutif et du Législatif, mais aussi au Ministère Public et aux particuliers ainsi qu'à l'ombudsman ${ }^{6}$. En d'autres termes, il démocratise le contentieux constitutionnel. Ceci est, en soi, assez significatif, compte tenu de l'état du droit comparé en la matière. L'ouverture est d'autant plus remarquable que la saisine par le particulier peut s'exercer aussi bien par voie d'action que par voie d'exception. ${ }^{7}$

En plus de son ouverture, le système de justice constitutionnelle instauré par la constitution du 18 mars 2005 est très attrayant pour le plaideur. La gamme des normes susceptibles d'être contrôlées (loi ordinaire, loi organique, loi référendaire, règlements autonomes, etc.) ainsi que l'étendue du bloc de constitutionnalité ou des normes de référence (texte de la constitution, instruments internationaux relatifs aux droits de l'homme) sont de nature à inciter le plaideur à saisir le juge constitutionnel au maximum. L'intérêt du plaideur à saisir le juge est d'autant plus grand que la déclaration d'inconstitutionnalité produit un effet radical. En effet, contrairement à ce qui s'observe dans beaucoup d'autres systèmes juridiques, la norme déclarée inconstitutionnelle en droit burundais sort définitivement de l'ordre juridique, et ceci quel que soit le mode par lequel le juge constitutionnel a été saisi. ${ }^{8}$

Conscient du contexte socioculturel dans et pour lequel il élaborait le système, le constituant de 2005 a, pour couronner le tout, enlevé quelques-uns des obstacles qui, au Bu-

du peuple burundais, source de son pouvoir et de son autorité. Le Gouvernement respecte la séparation des pouvoirs, la primauté du droit et les principes de la bonne gouvernance et de la transparence dans la conduite des affaires publiques. » (C'est nous qui soulignons).

5 Ce n'est pas, en réalité, la constitution du 13.3.1992 qui a instauré le multipartisme. La première constitution du Burundi indépendant (16.10.1962) avait instauré un système politique multipartite. Ce n'est qu'en date du 8.7.1966 que le monopartisme est instauré par l'arrêté royal n ${ }^{\circ} 001 / 2$ portant érection du parti UPRONA en parti unique national (B.O.B n ${ }^{\circ} .9 / 66$, p.315).

6 L'article 230 de la constitution dispose :

«La Cour Constitutionnelle est saisie par le Président de la République, le Président de l'Assemblée Nationale, le Président du Sénat, par un quart des membres de l'Assemblée Nationale ou un quart des membres du Sénat, ou par l'Ombudsman.

Toute personne physique ou morale intéressée ainsi que le Ministère Public peuvent saisir la Cour Constitutionnelle sur la constitutionnalité des lois, soit directement par voie d'action, soit indirectement par la procédure d'exception d'inconstitutionnalité invoquée dans une affaire soumise à une autre juridiction.

Celle-ci surseoit à statuer jusqu'à la décision de la Cour Constitutionnelle qui doit intervenir dans un délai de trente jours. ».

7 Article 230, al.2 de la constitution.

8 L'article 231, al. $1^{\mathrm{er}}$ de la constitution dispose : «Une disposition déclarée inconstitutionnelle ne peut être ni promulguée ni mise en application. » 
rundi plus qu'ailleurs, auraient empêché la saisine du juge constitutionnel par le particulier. Il a ainsi eu le (bon) reflexe de ne pas prévoir de délai de forclusion et de rendre la procédure gratuite 9 .

Tous ces ingrédients de la démocratisation du contentieux constitutionnel ne produiront cependant qu'un impact limité, dans les faits. La Cour constitutionnelle va d'abord se rendre difficilement atteignable par le citoyen en suivant une jurisprudence de 1992-1993 interprétant de manière extrêmement restrictive la notion d'intérêt à agir. Elle établira aussi un lien artificiel entre la qualité du saisissant et le type de norme attaquée en inconstitutionnalité, en déclarant que les particuliers ne peuvent la saisir que pour attaquer une loi, au sens strict du terme.

La thèse défendue par cet article est que le juge est allé à l'encontre de l'esprit de la constitution en restreignant de manière excessive l'accès des particuliers à la justice constitutionnelle. Cet article mettra d'abord en évidence le problème posé par ces restrictions (I). Il indiquera, dans un deuxième temps, pourquoi un revirement de jurisprudence s'impose et dans quel sens il devrait s'opérer(II).

\section{Les restrictions jurisprudentielles de l'accès des particuliers à la justice constitutionnelle}

Dans sa jurisprudence, la Cour constitutionnelle adopte une définition exagérément restrictive de la notion d' « intérêt à agir » (1). Elle établit, ensuite, un lien artificiel entre la qualité du requérant et le type de norme attaqué en inconstitutionnalité(2).

\section{L'interprétation ultra-restrictive de la notion d'intérêt à agir}

La Cour constitutionnelle suit toujours une jurisprudence des toutes premières années de sa création. ${ }^{10}$ En effet, c'est très tôt qu'elle eut à préciser les conditions dans lesquelles elle pouvait être saisie par les particuliers. Ceux-ci furent les premiers à la saisir; mises de côté, bien entendu, les saisines obligatoires du Président de la République en contrôle a priori de textes organiques. ${ }^{11} \mathrm{~A}$ des fins de clarté, il sied de d'abord présenter les arrêts ayant défini cette notion(A) avant de jeter un regard analytique sur l'influence intellectuelle qu'ils exercèrent sur les juges ultérieurs(B). L'on montrera, enfin, comment cette interprétation s'écarte de l'esprit de la constitution du 18 mars 2005 (C).

9 Article 17 de la loi $\mathrm{n}^{\circ} 1 / 018$ du 19 décembre 2002 portant Organisation et Fonctionnement de la Cour Constitutionnelle ainsi que la procédure applicable devant elle (telle que modifiée par la loi $n$ - $1 / 03$ du 11 janvier 2007).

10 RCCB 3 du 19.10.1992 (B.O.B. n 2/93, pp. 55-56) et RCCB 27 du 2 août 1993(inédit).

11 La première requête du particulier était le RCCB 3 du 19.10 .1992 (B.O.B. $n^{\circ}$ 2/93, pp. 55-56).Le RCCB1 du 13.4.1992 concernait le contrôle du décret-loi régissant la Cour constitutionnelle (texte organique) et le RCCB2 du 14. 4.1992, le contrôle du décret-loi sur les partis politiques (texte organique également). 


\section{A. La teneur des deux arrêts fondateurs : les RCCB 3 et RCCB 27}

En date du 19 octobre 1992, la Cour constitutionnelle fut saisie d'une requête d'un particulier demandant la déclaration d'inconstitutionnalité d'une disposition du Code d'Organisation et de la Compétence Judiciaires de l'époque qui attribuait au juge du Tribunal de Résidence le pouvoir d'exercer les fonctions du Ministère Public ${ }^{12}$ - et donc d'être juge et partie dans une même instance-. Elle déclara la requête irrecevable. Le requérant avait saisi le juge par voie d'action, c'est-à-dire en dehors de tout litige appelant application de la disposition attaquée. La Cour l'estima dépourvu de tout intérêt à agir. La juridiction constitutionnelle basait son raisonnement sur l'article 153 de la loi fondamentale du 13.3.1992-l'exact équivalent de l'article 230, al. 2 de la constitution de 2005-. Cette dernière disposition reconnait le droit d'action à toute "personne physique intéressée $»^{13}$ (c'est nous qui soulignons).

En bon pédagogue-comme tout juge de haut niveau devrait l'être-, la Cour se mit alors à expliquer le sens du groupe de mots «personne physique intéressée ». Elle précisa que la personne physique intéressée est une personne physique justifiant d'un intérêt personnel à agir, c'est-à-dire d'un intérêt qui lui est propre $»^{14}$. La juridiction constitutionnelle expliqua davantage son raisonnement en déclarant que, dans le contexte de l'article 153, seul le Ministère Public était habilité à agir en inconstitutionnalité dans un intérêt purement général. ${ }^{15}$

Selon la Cour dans ce même arrêt, l'intérêt propre doit, en outre, être un intérêt « juridiquement protégé ", c'est-à-dire un intérêt qui peut se justifier par référence à une règle de droit. Cet intérêt doit, enfin, être né et actuel. Résumant cette caractérisation de 1' «intérêt », la Cour déclara :

"Pour qu'une action en inconstitutionnalité émanant d'une personne physique soit recevable, celle-ci doit établir qu'elle a un intérêt personnel, né et actuel, et juridiquement protégé à agir devant la Cour. ${ }^{16}$

La Cour fera un raisonnement analogue à propos de la saisine par une personne morale lorsque, deux mois après les élections de juin 1993, elle sera saisie par le parti UPRONA en

12 L'article 8 de la loi n.1/004 du 14 janvier 1987 portant réforme de l'organisation et de la compétence judiciaires.

13 «Toute personne physique ou morale intéressée ainsi que le Ministère Public peuvent saisir la Cour constitutionnelle sur la constitutionnalité des lois, soit directement par voie d'action, soit indirectement par la procédure d'exception d'inconstitutionnalité invoquée dans une affaire soumise à une autre juridiction. Celle-ci surseoit à statuer jusqu'à la décision de la Cour constitutionnelle qui doit intervenir dans un délai de trente jours. ».

14 RCCB 3 du 19 octobre 1992, B.O.B. n²/93, p. 56.

15 RCCB 3 du 19 octobre 1992, B.O.B. n²/93, p. 56.

16 RCCB 3 du 19 octobre 1992, B.O.B. n²/93, p. 56. 
inconstitutionnalité de la $1 \mathrm{lo}^{17} \mathrm{n}^{\circ} 1 / 02$ du 15 décembre 1982 portant règlement intérieur de l'Assemblée Nationale. L'UPRONA demandait l'invalidation de ce texte dans sa totalité, ou, subsidiairement, de son article $2 .^{18}$

La Cour transposera les principes du RCCB 3 aux personnes morales, avec une légère nuance en ce qui concerne le caractère personnel de l'intérêt. Elle jugera que la personne morale doit justifier, "soit d'un intérêt propre, soit d'un intérêt directement en rapport avec son objet tel que défini par les lois pertinentes et les textes constitutifs de ladite personne morale.» ${ }^{19}$

Pour bien saisir le raisonnement de la Cour en ce qui concerne l'intérêt d'une personne morale, il est important de distinguer les sorts respectifs des deux éléments de la demande de l'UPRONA dans cette affaire. La requête en déclaration d'inconstitutionnalité de l'article 2 fut reçue par ce que la disposition attaquée limitait les possibilités de ce parti politique d'être représenté au bureau de l'Assemblée Nationale. La Cour estima alors, à juste titre, que 1'UPRONA défendait un « intérêt propre $»^{20}$. Par contre, la demande en déclaration d'inconstitutionnalité de toute la loi fut déclarée irrecevable pour défaut d'intérêt du requérant. Le parti UPRONA avait justifié son intérêt en faisant valoir qu'en tant qu'organisation politique soucieuse du fonctionnement régulier des institutions, elle avait intérêt à ce que l'Assemblée Nationale ne soit pas la première à violer les normes-en commençant par la plus importante- alors qu'une de ses missions importantes est justement de voter les lois. La Cour répondit que le fonctionnement régulier des institutions n'est, ni un intérêt propre, ni un intérêt directement en rapport avec l'objet social d'un parti politique. ${ }^{21}$ Dans les termes même de l'arrêt, le fonctionnement régulier des institutions n'était nullement un intérêt propre à l'UPRONA par ce que largement partagé par les autres forces politiques et sociales et même par la nation entière. ${ }^{22} \mathrm{Il}$ n'était pas davantage un intérêt directement en rapport avec l'objet social de l'UPRONA pour justifier son action dans la mesure où d'autres institutions, tels le Président de la République, le Premier Ministre, le Président de l'Assemblée Nationale et un quart des députés étaient qualifiées pour saisir la Cour, à cet effet. $^{23}$

La deuxième partie du raisonnement de la Cour est difficilement soutenable. En effet, s'il est très logique que le fonctionnement régulier des institutions n'est pas un "intérêt propre » à un seul parti politique, quel qu'il soit, il est de loin moins convaincant de soutenir qu'il n'entretient pas de rapports avec l'objet des partis politiques. L'objet d'un parti politique est, en effet, de chercher à conquérir le pouvoir; et ainsi de " faire fonctionner » les

17 Les règlements intérieurs des chambres du Parlement sont plutôt aujourd'hui des résolutions.

18 RCCB 27 du 2 août 1993.

19 RCCB 27 du 2 août 1993, quatrième feuillet, 2ème attendu, inédit.

20 RCCB 27 du 2 août 1993, sixième feuillet, 8ème attendu, inédit.

21 RCCB 27 du 2 août 1993, huitième feuillet, 3ème et 4ème attendu, inédit.

22 RCCB 27 du 2 août 1993, huitième feuillet, inédit.

23 RCCB 27 du 2 août 1993, huitième feuillet, inédit. 
institutions. D'un point de vue politique ensuite, il est difficilement défendable que dans un régime démocratique comme celui qu'instaurait la constitution du 13.3.1992, le contrôle du fonctionnement constitutionnel des institutions soit l'apanage des mêmes institutions. Enfin et surtout, en suivant cette ligne de raisonnement de la Cour, il n'y aurait plus aucune différence entre un «intérêt propre » et un « intérêt directement en rapport avec l'objet » d'une personne morale. Le propre du second est, en effet, de pouvoir être partagé, contrairement au premier dont l'essence est d'être exclusif. Le fait donc que, pour cette question, des institutions comme le Président de la République, le Premier Ministre, le Président de l'Assemblée Nationale, etc. étaient habilitées à saisir la Cour ne faisait nullement obstacle à ce que l'UPRONA puisse aussi la saisir.

Par ce manque de clarté, la Cour réduisait à peu de choses la portée pratique de son propre énoncé à savoir que les personnes morales peuvent agir pour défendre, non seulement un intérêt qui leur est propre mais aussi un intérêt directement en rapport avec leur objet. On verra dans la suite que presque plus aucune requête d'une personne morale ne sera reçue sur base d'un « intérêt directement en rapport avec l'objet». La Cour-dans ses différentes générations-demandera toujours la preuve d'un "intérêt propre.» ${ }^{24}$ Dans la pratique, il n'y avait donc plus aucune différence entre la saisine par un individu et la saisine par une personne morale.

Les arrêts RCCB 3 et RCCB 27 exercèrent une grande influence sur les différentes générations de la Cour. Leur essence est toujours suivie. Ils sont cependant, quelquefois, très mal interprétés et utilisés.

\section{B. La portée de l'influence des arrêts RCCB 3 et RCCB 27}

La Cour constitutionnelle du Burundi suit toujours la ligne tracée par les arrêts RCCB 3 et RCCB 27 dans l'appréciation de l'intérêt du requérant, lorsque celui-ci est un particulier, personne physique ou morale. Ceci fait que plusieurs dizaines de requêtes soient rejetées pour défaut d'intérêt de manière autrement discutable. Il serait matériellement impossible de revenir, dans cet article, sur toutes ces décisions. Un bref rappel du contenu de trois des arrêts parmi les plus emblématiques-et pas forcément des plus connus de l'opinion publique- suffira.

1. Le 16 avril 1994, la Cour déclara irrecevable, pour défaut d'intérêt, une requête en inconstitutionnalité d'un amendement de la constitution qui, du point de vue des requérants, menaçait la démocratie, la paix civile et la sécurité. ${ }^{25}$ Les requérants se débâtirent, en outre, pour démontrer que l'amendement qu'ils attaquaient prolongeait un pouvoir qui ne sécurisait pas la communauté ethnique à laquelle ils appartenaient mais la Cour trouva toujours cet intérêt trop « général » pour justifier la recevabilité d'une action d'un particulier. ${ }^{26}$

24 Voir infra, pp.7-8.

25 RCCB 39 du 18 avril 1994, B.O.B n ${ }^{\circ}$ 6/94, p.5.

26 RCCB 39 du 18 avril 1994, B.O.B n ${ }^{\circ}$ 6/94, p.5. 
2. Le 14 juin 2005, un parti politique saisit la Cour lui demandant de déclarer contraire à la constitution la loi portant Code électoral par ce que rédigée et votée en langue française alors que l'article 5, al.2 de la loi fondamentale pose le principe que «tous les textes législatifs doivent avoir leur version originale en kirundi.» ${ }^{27}$ Dans son arrêt RCCB126 du 11 juillet 2005, la Cour déclara la demande irrecevable. ${ }^{28}$ Le requérant eut beau plaider que, comme parti politique, il avait intérêt à ce que l'un des instruments juridiques les plus importants du jeu politique-le Code électoral-soit rédigé dans une langue que la plupart de ses militants comprennent, cela n'y fit rien. La Cour estima que le Code électoral est un texte de portée générale portant sur toutes les questions relatives aux élections et applicable à tous les acteurs politiques. Ce texte n'a donc pas, selon la Cour, de lien particulier avec un parti politique quelconque. Il ne suffit pas, toujours selon la Cour, d'alléguer que le texte promulgué en langue française a mis le parti requérant en situation défavorable, il faut montrer en quoi, par rapport à son objet et à ses textes constitutifs, la situation des militants de ce parti s'est trouvée défavorisée par le fait de la promulgation du Code électoral en langue française. ${ }^{29}$

Encore une fois, la Cour entretenait la confusion entre un intérêt propre et un intérêt en rapport avec l'objet de la personne morale. La Cour avait, sans le déclarer, exigé la preuve d'un intérêt exclusif. Sans cela, il deviendrait impossible de comprendre comment elle a pu estimer que le Code électoral-loi politique par excellence- n'a pas de lien particulier avec un parti politique. Le seul fait que d'autres partis politiques partagent le même intérêt n'aurait pas dû, en soi, empêcher que la demande du requérant soit reçue. En transposant ce raisonnement au droit social, c'est un peu comme si après un licenciement abusif ayant touché plusieurs salariés, le juge social refusait de recevoir l'action d'un travailleur licencié agissant seul au motif qu'il n'a pas été le seul à perdre son emploi. Le juge social aurait sûrement tort. La même chose peut être dite du juge constitutionnel burundais du 11 juillet 2005.

3. Le 27 juillet 2013 : La Cour déclare irrecevable une requête de la Maison de la Presse dirigée contre certaines dispositions de la loi $\mathrm{n}^{\circ} 1 / 11$ du 04 juin 2013 portant modification de la loi $\mathrm{n}^{\circ} 1 / 025$ du 27 novembre 2003 régissant la presse au Burundi. ${ }^{30}$ La Cour a estimé que cette organisation n'avait pas d' "intérêt personnel » à la saisir et qu'elle ne pouvait pas, non plus, agir au nom des professionnels des médias, par ce que non expressément mandatés par eux, ni dans ses statuts, ni dans une quelconque autre convention ad hoc. $^{31}$

Refuser à une « maison de la presse » le droit d'agir en justice constitutionnelle pour la défense de la liberté d'expression parait surprenant. Il est, en effet, évident que la défense

27 Codes et lois du Burundi (31 décembre 2006), p.5.

28 RCCB126 du 11.7.2005, inédit.

29 RCCB126 du 11.7.2005, inédit.

30 RCCB 269 du 27.6.2013, inédit.

31 RCCB 269 du 27.6.2013, inédit. 
de la liberté d'expression rentre dans l'objet social-explicite ou implicite- d'une quelconque organisation de journalistes.

Par l'arrêt Maison de la Presse, la Cour re-proclamait sa foi en cette jurisprudence vieille de plus de vingt ans selon laquelle il n'y a d'intérêt que l'intérêt propre. C'est d'ailleurs pour cette raison que, quatre mois plus tard, lorsqu'elle fut saisie par l'Union burundaise des journalistes(UBJ)-un syndicat de professionnels des médias-, elle trouva le requérant « intéressé » et reçut l'affaire. ${ }^{32} \mathrm{Il}$ y en a qui ont cru voir, en ce dernier arrêt, une réouverture de la Cour aux saisines des personnes morales défendant des causes altruistes. Rien de plus erroné. Dans sa motivation de l'arrêt UBJ, en effet, la Cour avait tenu à rappeler que conformément à sa «jurisprudence abondante », le requérant devait justifier d' "un intérêt qui lui est propre $»^{33}$.

Qu'il suffise cependant de rappeler que, selon la règle jurisprudentielle posée par l'arrêt RCCB 27-que la Cour affirme toujours suivre-, l'intérêt d'une personne morale agissant devant la Cour ne doit pas toujours être un intérêt propre. Il peut bien s'agir d'un intérêt " directement en rapport avec son objet tel que défini par les lois pertinentes et les textes constitutifs de ladite personne morale.»

Il est donc évident que dans sa pratique ultérieure aux arrêts RCCB 3 et RCCB 27, la Cour constitutionnelle ne s'est pas contentée d'appliquer à la lettre les règles posées par ces décisions-déjà très restrictives-. Elle a davantage restreint les possibilités de se faire saisir par les particuliers.

Sur un tout autre registre, la caractérisation de l'intérêt à agir pose également certains soucis pratiques à la Cour. La jurisprudence constante de la Cour constitutionnelle pose-très logiquement-que l'intérêt défendu doit être un intérêt « juridiquement protégé. » ${ }^{34}$ Dans les termes mêmes de la Cour, un intérêt est juridiquement protégé lorsqu'il peut se justifier par référence à une règle de droit. ${ }^{35}$ Les problèmes des moins attendus peuvent cependant surgir dans la compréhension de cette notion. A titre purement exemplatif, des difficultés sont apparues lorsque la Cour devait appliquer cette notion à une demande dirigée contre l'article 19 de loi organisant la Commission Terres et Autres et Biens(CNTB). ${ }^{36}$ Cet article dispose que les décisions de cette commission sont exécutoires nonobstant tout recours.

Pour justifier son intérêt à agir, le requérant-défendeur dans une affaire pendante à la CNTB-a soumis à la Cour que son droit à la propriété était en jeu. De manière assez surprenante, la Cour lui répondit que l'intérêt invoqué, à savoir le droit à la propriété n'était «nullement juridiquement protégé parce qu'il est aujourd'hui contesté par la partie adverse ${ }^{37} »$. Littéralement, la Cour a voulu dire que lorsqu'un droit est contesté par une autre partie-qui

32 RCCB 271 du 8 novembre 2013.

33 RCCB 271, deuxième feuillet, 5ième attendu.

34 Voir notamment le RCCB 3 du 19.10.1992 et le RCCB 27 du 2 août 1993.

35 RCCB 3 du 19.10.1992.

36 RCCB 256 du 9.3.2012.

37 RCCB 256 du 9.3.2012. 
peut être un particulier ou l'administration-, il cesse d'être juridiquement protégé et ne peut donc plus fonder la saisine de la Cour. Or, c'est justement lorsqu'un droit constitutionnel est contesté ou menacé que l'on a « intérêt » à agir en justice pour lui trouver une protection juridictionnelle. Dans cette affaire, c'est bien évidemment par ce que la propriété du requérant était contestée par une partie dans une instance pendante auprès de la CNTB que l'intérêt de saisir la Cour était né. Ici, l'intérêt du requérant était incontestablement protégé en droit, tout simplement par ce que le droit à la propriété qu'il est invoquait était consacré par la constitution. ${ }^{38} \mathrm{La}$ liquidation du contentieux sur le fond relevait d'une autre instance, et c'est justement l'existence de ce contentieux qui justifiait l'intérêt du requérant à saisir la juridiction constitutionnelle.

Ces restrictions jurisprudentielles de l'accès au juge constitutionnel sont difficilement compatibles avec l'esprit de la loi fondamentale.

C. La difficile compatibilité de l'interprétation jurisprudentielle de la notion d'intérêt avec l'esprit de la constitution de 2005.

Dans son arrêt RCCB 3, la Cour admit baser sa compréhension de la notion d'intérêt sur "le droit commun burundais.» ${ }^{39}$ En d'autres termes, par rapport à la notion d'intérêt, le juge constitutionnel a considéré le contentieux normatif comme le contentieux de droit commun. La disposition législative pertinente en droit commun est l'article 3 du Code de Procédure Civile. ${ }^{40} \mathrm{C}$ 'est donc des règles du contentieux de droit privé que la Cour a tiré sa conception de la notion d'intérêt à agir en droit constitutionnel. Le premier problème avec cette façon de voir est que le contentieux de droit constitutionnel n'est pas du tout comparable au contentieux de droit privé, en ce qui concerne l'intérêt à agir. Le deuxième problème est que les travaux préparatoires de la constitution du 13 mars 1992 ainsi que la version kirundi de la disposition pertinente de cette même constitution (l'article 153) conduisaient logiquement à une conclusion différente de celle à laquelle a abouti le juge de 1992.

\section{a) La particularité de la notion d'intérêt à agir en droit constitutionnel}

Les règles du droit constitutionnel ne s'interprètent pas comme celles du droit privé. Les arguments en faveur d'une spécificité des techniques interprétatives sont généralement au nombre de trois. D'une part, la constitution est composée de principes qui n'admettent pas une lecture littérale mais doivent être interprétés; d'autre part, les antinomies entre les principes constitutionnels ne peuvent être résolues à l'aide des critères classiques mais doivent prendre la forme d'une pondération, d'un balancement entre principes; enfin, le juge consti-

38 Article 36 de la constitution, in Codes et lois du Burundi, Tome I (31decembre 2006), p.6.

39 RCCB 3 du 19 octobre 1992, B.O.B n 2/93, p.56, deuxième attendu.

40 «L'action est ouverte à tous ceux qui ont un intérêt légitime. Elle n'est reconnue qu'aux seules personnes capables d'ester en justice et qualifiées par la loi pour élever ou combattre une prétention. » (Codes et lois du Burundi, Tome II, p.208). 
tutionnel se situe à mi-chemin entre le législateur et le juge ordinaire : il est libre comme peut l'être le législateur mais cette liberté est encadrée par des exigences prudentielles, ce qui l'éloigne du juge ordinaire qui, lui, est tenu de se conformer au modèle de la subsomption. ${ }^{41}$

Plus spécifiquement, en ce qui concerne la notion d'intérêt à agir, l'idée, en droit privé, est de n'ouvrir le prétoire qu'au véritable titulaire du droit. La raison en est toute simple : il faut éviter que ce dernier ne soit préjudicié par le zèle d'un plaideur sans intérêt légitime.

Gallet, L. l'exprime très clairement lorsqu'il écrit que «la qualité pour agir...n'est que la traduction processuelle de la titularité du droit substantiel. $»^{42}$ En droit commun, la qualité pour agir présente donc toujours un lien avec le droit subjectif allégué par le demandeur. Il s'agit notamment du droit à la réparation du dommage subi. Il s'impose alors, toutà-fait logiquement, de veiller à ce que les prestations dues soient octroyées à leur véritable créancier. Or, dans un contentieux normatif-de constitutionnalité spécifiquement-, le requérant ne défend jamais, en principe, un intérêt qui lui est exclusif. En effet, sa cible est une norme-une loi généralement-et par définition, la loi est générale. S'il est incontestable qu'elle peut affecter un individu ou un groupe d'individus dans ses intérêts beaucoup plus significativement qu'un autre, personne n'est fondé à soutenir que la loi le concerne seul, qu'elle est, pour ainsi dire, la sienne. Il s'en suit que personne ne peut soutenir avoir un droit exclusif à contester sa validité constitutionnelle. L'exigence de l'intérêt dans le contentieux de droit privé répond donc à un impératif absent dans le contentieux de droit public-et de droit constitutionnel notamment-.

Dans l'affaire $R v$ Somerset County Council and ARC Southern Limited ex p Dixon, Sedley, J.a bien résumé la différence essentielle existant entre le droit public et le droit privé et les implications que cela doit avoir, en ce qui concerne l'intérêt et la qualité à agir en justice. Pour cet illustre juge, alors que le droit privé concerne essentiellement des droits (subjectifs), le droit public concerne davantage des abus de droits. Il en résulte que pour le contentieux de droit public, les juridictions doivent moins se préoccuper de la qualité et de l'intérêt du requérant, d'autant plus que ce dernier peut agir pour le bien commun. ${ }^{43} \mathrm{Ce}$ raisonnement n'a rien de typiquement anglo-saxon. Il est donc également valide dans d'autres familles juridiques reconnaissant la différence entre les deux types de contentieux.

41 Brunet, P.: Le juge constitutionnel est-il un juge comme les autres? Réflexions méthodologiques sur la justice constitutionnelle in Grewe, C., Jouanjan, O., Maulin, E. \& Wachsmann, P. (ed.) : $L a$ notion de justice constitutionnelle, Dalloz, Paris, 2005, pp. 115-135.

42 Gallet, J.L : L'accès à la justice dans les traditions constitutionnelles nationales - La qualité pour agir dans l'ordre juridique français (document du séminaire de l'ERA portant sur « L'accès à la justice dans le droit communautaire : le rôle du juge national », organisé en coopération avec la Cour de cassation les 1 er et 2 juin 2006 à Paris.).

43 "Public law is not at base about rights, even though abuses of power may and often so invade private rights: it is about wrongs-that is to say misuses of public power; and the courts have always been alive to the fact that a person or organization with no particular stake in the issue or the outcome may, without in any sense being a mere meddler, wish and be well placed to call the attention of the court to an apparent misuse of public power.". 
Le même raisonnement est suivi par plusieurs juridictions africaines dont la Court of Appeal du Botswana. Celle-ci a déclaré, notamment dans l'affaire Attorney General v. Dow que, dans la mise en œuvre de libertés constitutionnelles, la qualité d'agir ne doit pas être refusée au requérant sur des bases étroitement techniques. ${ }^{44}$ De la même manière et selon la même jurisprudence, la requête ne doit pas être freinée par des filtres procéduraux dérivés des principes généraux de la Common Law, du droit romano-germanique ou d'un quelconque autre système juridique. ${ }^{45} \mathrm{~L}$ 'on admet, de plus en plus, qu'un système juridique qui, sur base de règles étroitement techniques de qualité pour agir, empêcherait aux individus et aux groupes de pression altruistes d'agir en justice pour la défense de l'état de droit, serait gravement lacunaire. ${ }^{46}$

Ce raisonnement est beaucoup plus usité dans le contentieux des libertés publiques et des droits de l'homme. C'est par ailleurs le même raisonnement que suit la commission africaine des droits de l'homme et des peuples. ${ }^{47}$ Dans plusieurs des communications qu'elle reçoit, la commission est saisie par des ONGs œuvrant dans le domaine des droits de l'homme, sans même tenir compte du fait qu'elles sont ou non enregistrées comme personnes juridiques dans l'un quelconque des Etats parties. Il n'est même pas requis que les organisations requérantes soient mandatées par les supposées victimes des violations de la charte. ${ }^{48}$

b) Les travaux préparatoires de la constitution du 13 mars1992 et la version Kirundi de l'article 153 de cette dernière en opposition avec les arrêts RCCB 3 et RCCB 27.

Le juge de 1992-RCCB 3-avait fait recours aux travaux préparatoires de la Constitution. Il soupçonnait déjà que le constituant ait pu donner à l'expression "personne physique intéressée » un sens différent de celui du droit commun. A son examen du «Rapport sur la démocratisation des institutions et de la vie politique au Burundi d'août 1991»-document valant travaux préparatoires de la constitution du 13mars1992-, la Cour y trouva tout simplement l'expression «toute personne physique». Il en tira alors la conclusion que c'était le constituant qui avait « ajouté » à l'expression le qualificatif « intéressée » dans son texte

44 Viljoen, F. International human rights law in Africa, Oxford- University Press, oxford p.324.

45 «Constitutional rights should not be whittled down by principles derived from the common law, the Roman-Dutch, English or Botswana. And (...) standing should not be denied on purely technical grounds".

46 Tafur Isabella écrit notamment ceci : ..... «It would, to paraphrase Lord Diplock, be a grave lacuna in our system of public law if a pressure group or single public-spirited individual were prevented by technical rules on standing from bringing unlawful conduct to the attention of the court to vindicate the rule of law and get the unlawful conduct stopped ( $R v$ Inland Revenue Commissioners, ex parte National Federation of Self-employed and Small Businesses Ltd [1982] AC 617).

47 Pour plus de détails, voir notamment: Christensen, C. \& Odinkalu, C.A.: The African Commission on Human and Peoples' Rights, Human Rights Quarterly, vol.20, pp.235-280.

48 Viljoen, F. op. cit, p. 324. 
final, et ceci à des fins de précision. Ce qualificatif devait alors nécessairement avoir, selon la même Cour, une « signification juridique », lisez : de droit commun.

C'est la conclusion inverse qu'il fallait tirer, à notre avis. En effet, si les travaux préparatoires parlent de « toute personne physique» et que rien n'indique pourquoi le qualificatif « intéressée » a été ajouté au texte final de la constitution, il faut en déduire que ce qualificatif doit plutôt être compris dans son sens commun et ordinaire et ne pas chercher à lui attribuer une quelconque signification technique. Cette approche est d'autant plus fondée que c'est le sens commun-et non juridique- de ce qualificatif qui est conforme à la version kirundi de l'article 153 de la constitution de 1992 comme vont le montrer les lignes qui suivent.

En outre, la recherche de l'intention du constituant ne doit pas se confondre avec la recherche du sens des mots. Elle vise plutôt à découvrir la "valeur fondamentale » véhiculée par une disposition. ${ }^{49}$ Par l'article 153 de la constitution de 1992 et plus tard par l',article 230, al. 2 de celle de 2005, il est évident que le constituant voulait encadrer, autant que faire se pouvait, l'action du parlement pour la préservation de l'état de droit. C'est pour cela qu'il avait multiplié au maximum les occasions de censure du législateur. Rien d'autre n'explique que le droit de saisine ait été accordé, non seulement aux autorités et institutions politiques, mais aussi aux particuliers intéressés et au Ministère Public. Il est aussi évident que c'est pour arriver à cet objectif que le constituant avait fait abstraction de la catégorisation juridique classique et donné le droit de saisine à un quart des représentants. En effet, un groupe de députés ou de sénateurs-quelle que soit sa taille- n'est ni une personne juridique, ni un organe de l'Etat. En droit judiciaire classique, il n'y aurait pas de possibilité de lui ouvrir les portes du prétoire, sauf comme ensemble de plaignants individuels. Or, ce n'est pas de cette dernière manière que l'article 153 de la constitution du 13 mars 1992-équivalent de l'actuel article 230-envisageait la saisine de la Cour constitutionnelle par un quart de représentants.

Il subsiste cependant une question : comment comprendre le qualificatif " intéressé » s'il doit avoir un sens différent de celui que lui confère l'arrêt RCCB 3?

Sans soutenir qu'une version linguistique du texte constitutionnel ait plus de foi qu'une autre $^{50}$, il semble que la version kirundi de l'article 153 de la constitution de 1992 rend mieux compte de l'esprit de la constitution. En effet, dans le texte kirundi, l'expression "personne physique intéressée » est rendue par "umuntu uwo ari wese abikeneye ». De même, "personne morale intéressée » est rendue par " ishirahamwe iryo ari ryose ribikeneye ». Littéralement, ces deux dernières expressions kirundi veulent respectivement dire : «personne physique qui en éprouve le besoin » et «personne morale qui en éprouve le besoin ». Le besoin est certainement différent de l' "intérêt » tel qu'appréhendé par le droit commun. En effet, alors que ce dernier est objectif, vérifiable et discutable par la partie ad-

49 Kenneth, R.T: Selected theories of constitutional interpretation. Congress Research Service. February, 15, 2011, pp.6-7.

50 Voir pour cela l'arrêt RCCB 7 du 7 décembre 1992, 2ème à 4ème feuillet. Inédit. 
verse, le « besoin » est, par essence, subjectif et insusceptible de remise en cause externe. Du simple fait d'avoir bougé de chez elle pour se diriger vers le palais, la personne qui saisit le juge pour contester la validité constitutionnelle d'une norme doit être considérée comme en ayant absolument besoin. Elle doit alors, aux termes de l'article 230, al. 2, être considérée comme y étant « intéressée ». L'on ne peut que lui empêcher d'abuser de la procédure si l'on a des raisons légitimes d'estimer qu'il est de mauvaise foi.

L'expression kirundi qui rend compte du concept d'intérêt en droit commun est « inyungu». Si, pour agir devant la juridiction constitutionnelle, le constituant de 1992 avait voulu exiger un intérêt comparable à celui du droit commun, il aurait, en Kirundi, dit: " umuntu wese abifisemwo inyungu » et « ishirahamwe ryose ribifisemwo inyungu ».

Il existe, en droit comparé, des juridictions constitutionnelles devant lesquelles un intérêt similaire à celui requis en droit privé doit être prouvé. Conscients de déroger à un principe plutôt général, les rédacteurs des constitutions dans ces pays prennent alors le soin de le formuler le plus clairement possible. Ainsi, par exemple, l'article 142, 3 de la constitution belge précise que la Cour constitutionnelle de Belgique ne peut être saisie que par une série d'autorités que la loi désigne, les juridictions à titre préjudiciel ainsi que les particuliers «justifiant d'un intérêt». Le constituant burundais ne l'ayant pas envisagé comme tel, l'on ne peut qu'en déduire qu'il n'a pas voulu exiger la preuve d'un intérêt comparable à celui requis en droit commun.

Cette interprétation ne doit pas être considérée comme déraisonnable. Il existe, en effet, en Afrique, des juridictions constitutionnelles qui ne requièrent la preuve d'aucun intérêt du tout pour recevoir les requêtes qui leur sont adressées. La Cour constitutionnelle du Bénin en est un exemple. Elle a déjà reçu des demandes des particuliers, même dans le contentieux politico-institutionnel, sans requérir la preuve d'un quelconque intérêt. Ainsi, par exemple, en date du 5 avril 1996, elle a reçu et donné suite à une requête de Monsieur FAGNINOU Gilles R.B qui se plaignait du non respect de la constitution par le Président de la République lors de sa prestation de serment. Fervent chrétien, le Président élu avait sciemment omis une partie du serment qu'il considérait comme inspirée de l'animisme. ${ }^{51} \mathrm{Il}$ est certain que si Monsieur FAGNINOU avait été Burundais agissant devant la C.C. burundaise, il n'aurait reçu de « sa » cour qu'un énergique « de quoi tu te mêles? »

En conclusion de ce point consacré à l'intérêt à agir, il est évident que le juge de 1992 a mal interprété la disposition pertinente -l'article 153- de la constitution de l'époque. En conséquence, le juge qui interprète la constitution du 18 mars 2005 a tort de le suivre.

L'exigence de l'intérêt né, actuel et juridiquement protégé n'est pas le seul obstacle à l'accès au juge constitutionnel par les particuliers. Le lien entre la qualité du saisissant et le type de norme attaqué en est un autre.

51 Décision DCC-96-017 du 5 avril 1996. (www.cour-constitutionnelle-benin.org). 
2. Le lien artificiel créé entre la qualité du requérant et le type de norme attaqué

L'article 230 classe en deux catégories les personnes habilitées à saisir la juridiction constitutionnelle. En son alinéa 1 ${ }^{\mathrm{er}}$, il dispose que la C.C est saisie par le Président de la République, le Président de l'Assemblée Nationale, le Président du Sénat, un quart des membres de l'Assemblée Nationale ou un quart des membres du Sénat, ou par l'Ombudsman. Il n'est fait aucune précision sur la matière pour laquelle ces autorités peuvent saisir la Cour. L'on en déduit qu'elles peuvent saisir cette dernière pour l'exercice de l'une quelconque des compétences énumérées à l'article 228(contrôle de constitutionnalité, interprétation, constat de vacance du président, etc.).

L'alinéa 2 du même article reconnait, quant à lui, à toute personne physique ou morale et même au Ministère Public le droit de saisir la Cour mais, à la différence de l'alinéa ${ }^{\text {er }}$, il précise la compétence pour laquelle ils saisissent la Cour : le contrôle de la constitutionnalité des lois.

Il existe donc un lien voulu par le constituant lui-même entre la qualité de la personne qui saisit la Cour et la compétence matérielle de cette dernière. Ainsi, alors que les autorités énumérées à l'article 230 , al. $1^{\mathrm{er}}$ peuvent la saisir pour l'exercice de l'une quelconque des compétences énumérées à l'article 228, les particuliers, ainsi que le Ministère Public, ne peuvent saisir la Cour que pour le contrôle de constitutionnalité, première compétence de cette juridiction posée au premier trait de l'article 228 de la constitution. Il en résulte que, pour l'exercice des autres compétences (interprétation de la constitution, constat de vacance du poste de Président, etc..), la Cour ne saurait être saisie par un particulier. L'une ou l'autre des autorités énumérées à l'article 230 , al. $1^{\mathrm{er}}$ doit vouloir bouger. La solution parait justifiée. Il est logique que la saisine pour l'exercice d'une compétence touchant essentiellement à la vie politique de l'Etat soit réservée aux institutions politiques. Les particuliers ne sont, de prime abord, intéressés à l'ordre constitutionnel que lorsque leurs libertés sont en jeu et c'est à cela que répond l'existence de leur droit de saisine en contrôle de constitutionnalité.

Beaucoup moins convaincante est cependant l'interprétation que fait la Cour du concept de «loi » contenu dans l'alinéa 2 de l'article 230. Lorsque la Constitution dispose que toute personne intéressée ainsi que le Ministère Public peuvent saisir la Cour sur la constitutionnalité des lois, la Cour lit le concept loi dans son sens strict et technique voulant dire norme d'origine parlementaire. ${ }^{52}$ Elle en déduit que le particulier ne peut saisir la Cour du contrôle d'un règlement autonome. ${ }^{53}$ Or, l'on sait que l'article 228 qui règle la compétence de la Cour constitutionnelle dispose, en son premier trait, que la Cour est compétente pour contrôler les « lois et actes réglementaires pris dans les matières autres que celles relevant $d u$ domaine de la loi.» ${ }^{54} \mathrm{La}$ lecture littérale combinée de ces deux dispositions conduit à la

52 Voir notamment l'arrêt RCCB 174 du 22/8/2006, in B.O.B n6bis/2012, pp. 1053-1054.

53 Voir notamment l'arrêt RCCB 174 du 22/8/2006, in B.O.B n6bis/2012, pp. 1053-1054.

54 Codes et lois (31 décembre 2006), Tome I, p.18. 
conclusion que, même s'il est indiscutable que la Cour est habilitée à contrôler les règlements autonomes, elle ne peut le faire que sur saisine des institutions et autorités énumérées à l'article 230, al. $1^{\text {er }}$ (Président de la République, Président de l'Assemblée Nationale, Président du Sénat, un quart des députés ou sénateurs et l'ombudsman).

Il est cependant plus raisonnable d'estimer que, par la précision apportée à l'alinéa 2 de l'article 230 (contrôle de la constitutionnalité des lois), le constituant a seulement voulu exclure la saisine de la Cour par les particuliers pour l'exercice des compétences autres que le contrôle normatif(interprétation, constat de vacance du poste de vice-président,....). Rien ne semble indiquer que la volonté ait été d'exclure le droit des particuliers de déclencher le contrôle des règlements autonomes.

L'interprétation contraire manquerait de sens, même d'un point de vue politique. Dans un système démocratique, si le citoyen peut faire contrôler l'action de son mandataire et représentant direct (le Parlement), peut-on légitimement lui refuser le moindre regard sur l'action normative du Président de la République dans les domaines où celui-ci agit en toute indépendance par rapport au Parlement (règlements autonomes)?

En outre, du point de vue des exigences de l'état de droit, qu'est-ce qui justifierait que le citoyen soit démuni devant un risque d'empiétement de ses droits par des actes insusceptibles du contrôle de légalité? En effet, les décrets fondés sur l'article 160 de la constitution ne sont susceptibles d'aucun contrôle de légalité par une juridiction administrative, tout simplement par ce que ces règlements n'exécutent aucune législation. Ils sont en dehors du domaine de la loi. Ils ne sont susceptibles que du contrôle de constitutionnalité.

Raisonnablement donc, le terme « loi » utilisé par l'article 230, al. 2 est à comprendre dans son sens large, dans les limites, bien entendu, de la compétence matérielle de la Cour constitutionnelle en matière de contrôle normatif, tel que posé par l'article 228, premier trait de la constitution. Il s'ensuit que le particulier peut saisir la Cour constitutionnelle du contrôle des «lois et actes réglementaires pris dans les matières autres que celles relevant du domaine de la loi. ».

La Cour semble cependant avoir adopté l'interprétation littérale ci-haut donnée, bien malgré son absurdité apparente. Soulignons en passant qu'elle se trompe aussi des fois, à notre avis, sur le sens du concept de règlement autonome. Tel a notamment été le cas dans son arrêt RCCB 174. Dans cette affaire, la Cour a jugé que des organisations non gouvernementales (LIGUE ITEKA, OAG, FORSC) manquaient de qualité pour la saisir d'une requête dirigée contre deux ordonnances du Ministre de la Justice. L'objet de ces deux ordonnances était l'" élargissement provisoire » (sic) des prisonniers politiques détenus dans les maisons de détention de la République du Burundi. La Cour a justifié sa décision en indiquant que l'acte attaqué était un règlement et que pour cette raison, les particuliers ne pouvaient l'en saisir par ce qu'ils ne sont habilités à saisir la Cour qu'en contrôle de constitutionnalité des lois. ${ }^{55} \mathrm{La}$ Cour y a donc vu un problème de saisine et non de compétence matérielle. Elle n'aurait cependant pu aboutir à cette conclusion qu'après avoir considéré ces 
ordonnances comme des règlements autonomes. Si la Cour s'était bien rendu compte de la véritable nature juridique de ces ordonnances-règlement d'exécution ${ }^{56}$-, la solution logique aurait été de se déclarer matériellement incompétente. La Cour ne contrôle pas, en effet, la constitutionnalité des règlements d'exécution. En vertu de l'article 228, premier trait, de la constitution, les normes susceptibles d'un contrôle de constitutionnalité sont seulement les lois et actes réglementaires pris dans les matières autres que celles relevant du domaine de la loi. Bien malgré cette erreur, la Cour avait montré sa rigidité dans l'interprétation du concept de loi relativement aux exigences de la saisine posées par l'article 230, al. 2 de la constitution.

Dans certains textes constitutionnels ayant précédé celui du 18 mai 2005, les dispositions étaient relativement plus claires. Il en est ainsi de la constitution du 13 mars 1992 et de la constitution de transition du 28 octobre 2001.

Dans la constitution de 1992, les dispositions pertinentes étaient les articles 151 et 153. L'article 151, premier trait, posait le principe que la Cour était compétente pour contrôler la constitutionnalité des lois et des règlements autonomes, exactement comme le fait l'article 228 , premier trait de la constitution de 2005. Il précisait cependant également que la Cour exerçait cette compétence sur saisine aussi bien des autorités publiques (Président de la République, Premier Ministre,..) que des particuliers et du Ministère Public. ${ }^{57} \mathrm{Il}$ est évident que cet article réglait, en même temps, deux questions : la compétence et la saisine. Du point de vue de la légistique, cette rédaction est plutôt inhabituelle. Elle est même relativement maladroite dans la mesure où une partie de la disposition qui réglait la saisine par les particuliers -l'article $153^{58}$-devenait redondante. Il est intéressant de relever que ce dernier article utilisait exactement les mêmes expressions que l'actuel article 230, al.2. Il parlait de la saisine « en inconstitutionnalité de la loi ${ }^{59}$ (C'est nous qui soulignos). Il ne reprenait donc pas la fastidieuse formule de l'article 151 : "lois et actes règlementaires pris dans les matières autres que celles relevant du domaine de la loi ». Personne n'a cependant prétendu

56 Les ordonnances attaquées mettaient, en effet, en œuvre, le décret n ${ }^{\circ} 100 / 02$ du 03 janvier 2006 portant immunité provisoire des prisonniers politiques détenus dans les maisons de détention de la République du Burundi (B.O.B n²1/2006).

57 «....Statuer sur la constitutionnalité des lois et des actes règlementaires pris dans les matières autres que celles relevant du domaine de la loi sur demande du Président de la République, du Premier Ministre, du Premier Ministre, du Président de l'Assemblée Nationale, d'un quart des représentants ou des personnes et de l'organe vises à l'article 153...».

58 Toute personne physique ou morale intéressée ainsi que le Ministère Public peuvent saisir la Cour Constitutionnelle sur la constitutionnalité des lois et des actes réglementaires pris dans les matières autres que celles relevant du domaine de la loi, soit directement par voie d'action, soit indirectement par la procédure d'exception d'inconstitutionnalité invoquée dans une affaire soumise a une autre juridiction. Celle-ci surseoit à statuer jusqu' à la décision de la Cour Constitutionnelle qui doit intervenir dans un délai de 30 jours. » (B.O.B 10/2001, p.1293).

59 Loc cit. 
que les articles 151 et 153 se contredisaient. Dans la constitution de transition du 28 octobre 2001, la disposition pertinente était l'article 185, al.2. ${ }^{60}$

C'est donc à des fins de bonne légistique constitutionnelle que le constituant de $2005 \mathrm{a}$ adopté des formules plus courtes. C'est pour la même raison que l'actuel article 228, premier trait, ne désigne plus les personnes et institutions habilitées à la saisir, comme le faisait l'article 151 de la constitution de 1992. Le constituant choisit, en effet, de réserver à la saisine une disposition entière et séparée- l'article 230-. Cette interprétation nous parait d'autant plus défendable que rien-et vraiment rien-dans les travaux préparatoires de la constitution du 18.5.2005 ne parait suggérer une quelconque volonté du constituant de réduire l'étendue des matières pour lesquelles le particulier et le Ministère Public peuvent saisir la cour constitutionnelle.

Au-delà de la jurisprudence, la supposée contrariété entre les articles 228 et 230 fut à l'origine d'une révision de la loi organisant la procédure applicable devant la cour constitutionnelle. ${ }^{61}$

L'interprétation aussi réductrice du concept de « loi » ne se rencontre généralement pas dans les pratiques d'autres juridictions constitutionnelles. Ainsi, par exemple, alors que l'article 122 de la constitution béninoise est libellé exactement de la même manière que l'article 230, al.2 de la constitution burundaise et ne parle donc que de saisine des particuliers pour le contrôle de la constitutionnalité des lois, la Cour comprend ce concept dans son sens général de toute norme de droit écrit. Elle reçoit ainsi les requêtes de citoyens dirigées contre des lois, des règlements et autres actes présumés inconstitutionnels. ${ }^{62}$

Un revirement de jurisprudence du juge burundais s'impose donc, en conséquence.

\section{Un nécessaire revirement de jurisprudence : sensibilité au constitutionnalisme (moderne) et meilleure prise en compte du contexte politique burundais.}

Les lignes qui précèdent auront démontré que la Cour constitutionnelle du Burundi s'est juridiquement distancée de l'esprit-et quelquefois de la lettre-de la constitution en interprétant de manière ultra-restrictive les dispositions régissant l'accès au juge constitutionnel. Dans les lignes qui suivent, l'on montrera que cette interprétation se situe en marge du constitutionnalisme moderne, ou du constitutionnalisme tout court. Le contentieux constitutionnel moderne réserve, en effet, une place importante aux droits et libertés. Ceux-ci ne peuvent

60 «....Toute personne physique ou morale intéressée ainsi que le Ministère Public peuvent saisir la Cour Constitutionnelle sur la constitutionnalité des lois et des actes réglementaires pris dans les matières autres que celles relevant du domaine de la loi, soit directement par voie d'action, soit indirectement par la procédure d'exception d'inconstitutionnalité invoquée dans une affaire soumise à une autre juridiction. Celle-ci surseoit à statuer jusqu' à la décision de la Cour Constitutionnelle qui doit intervenir dans un délai de 30 jours. » (B.O.B 10/2001, p.1293).

61 Loi $\mathrm{n}^{\circ} 1 / 03$ du 11 janvier 2007 portant modification de certaines dispositions de la loi $\mathrm{n}^{\circ} 1 / 018 \mathrm{du}$ 19 décembre 2002 portant Organisation et Fonctionnement de la Cour Constitutionnelle ainsi que là procédure applicable devant elle.

62 BADET, S.G., op.cit, p.170. 
être bien protégés que dans le cadre d'une juridiction ouverte aux victimes actuelles et éventuelles de leurs violations ainsi qu'à leurs porte-voix(1). Au demeurant, les restrictions de l'accès au juge constitutionnel ne répondent, dans le cas du Burundi, à aucun besoin pratique. Contrairement à ce qui s'observe dans beaucoup d'autres pays africains, la Cour constitutionnelle burundaise est bien loin d'être surchargée. Le contexte sociopolitique burundais fait aussi que seuls les particuliers sentent le besoin de saisir la Cour. Leur fermer la porte de la justice constitutionnelle équivaut ainsi à condamner cette dernière à la non-existence (2).

1. L'importance des droits de l'homme dans le contentieux constitutionnel moderne et la position du constituant de 18 mars 2005.

Les droits de l'homme constituent la partie la plus importante du droit constitutionnel moderne. Selon Kanté, B., le droit constitutionnel classique revêtait deux dimensions : une partie consacrée à la théorie générale et une autre consacrée aux aspects institutionnels. Le droit constitutionnel moderne, en revanche, qui correspond à la naissance du constitutionnalisme, ajoute deux nouvelles dimensions: les droits et libertés fondamentaux d'une part, la justice constitutionnelle d'autre part. ${ }^{63}$ Les droits de l'homme constituent ce que le même auteur appelle «le droit constitutionnel relationnel». ${ }^{64}$ Cette branche du Droit devient progressivement la plus importante dans les constitutions africaines et le Burundi s'intègre de plus en plus dans la même logique. La justice constitutionnelle doit ainsi évoluer dans le même sens.

Il est évident que le constituant burundais a envisagé un lien fort entre droits de l'homme et contrôle de constitutionnalité. En témoigne le libellé de l'article 48 de la constitution :

"Les droits fondamentaux doivent être respectés dans l'ensemble de l'ordre juridique, administratif et institutionnel. La Constitution est la loi suprême. Le législatif, l'exécutif et le judiciaire doivent la faire respecter. Toute loi non conforme à la Constitution est frappée de nullité. $\|^{65}$

Depuis 1992, la protection des droits de l'homme était reconnue comme étant le fondement principal du droit de saisine de la C.C. reconnu aux particuliers. La commission constitutionnelle le reconnaissait sans ambages en ces termes :

«Il convient de signaler (...) que le contrôle de constitutionnalité inclut bien évidemment les lois et les règlements qui seraient en contradiction avec les droits de l'homme proclamés par la Constitution. C'est surtout au regard de telles situations

63 Kanté, B. op.cit, p. 241.

64 Ibidem, p. 241.

65 Codes et lois du Burundi (décembre 2006), Tome I, p.7. 
que la Commission a proposé que les individus puissent saisir la Courconstitutionnelle.... $»^{66}$

Plus évocatrice du lien voulu entre la protection des droits de l'homme et le contentieux constitutionnel est cependant la deuxième mission confiée à la juridiction constitutionnelle dans le texte du 18 mars 2005: « assurer le respect de la présente Constitution, y compris la Charte des droits fondamentaux, par les organes de l'Etat et les autres institutions ». Il s'agit d'une compétence totalement distincte et indépendante du contrôle de la constitutionnalité des lois, cité au premier trait de ce même article 228.

En droit comparé, il est aussi évident que c'est la préoccupation des droits humains qui justifie sans doute la consécration du recours individuel en matière de contrôle abstrait. ${ }^{67}$ Un exemple parmi des dizaines est l'évolution de la justice constitutionnelle belge en cette matière. En droit belge, le contrôle abstrait a été utilisé comme l'affaire des politiques jusqu'en 1989. Il ne s'agissait, par cette voie, que de défendre les grands équilibres constitutionnels, les particuliers se contentant du recours individuel concret, c'est-à-dire par voie d'exception. A partir de 1989, le contrôle abstrait a été étendu aux particuliers. L'on s'est, en effet, aperçu que les questions nouvelles à trancher par le juge constitutionnel allaient avoir, au-delà du respect des équilibres constitutionnels, un impact direct sur la sauvegarde des droits fondamentaux individuels. ${ }^{68} \mathrm{La}$ juridiction constitutionnelle ne pouvait alors rester une simple Cour d'arbitrage des conflits entre organes constitutionnels. ${ }^{69}$

Dans le cas précis du Burundi, il se pose la question de savoir la manière dont la Cour constitutionnelle doit mettre en œuvre l'article 228, deuxième trait, c'est-à-dire exercer la compétence consistant à : " assurer le respect de la présente Constitution, y compris la Charte des droits fondamentaux, par les organes de l'Etat et les autres institutions.» ${ }^{70} \mathrm{~A}$ titre de rappel, il s'agit d'une compétence totalement différente du contrôle de la constitutionnalité des lois. La question se pose alors en termes suivants : comment assure-t-on le respect d'une constitution (de l'une quelconque de ses dispositions, plus exactement), y compris sa charte de droits fondamentaux, autrement que par le contrôle normatif de constitutionnalité? Dans le cas du Burundi, la difficulté est d'autant plus grande qu'aucune autre règle, ni constitutionnelle, ni législative, encore moins règlementaire, n'indique comment cette compétence doit être exercée. La constitution du 18 mars 2005 est muette à ce sujet, de même d'ailleurs que la loi de 2002 relative à l'organisation et au fonctionnement de la Cour Constitutionnelle, telle que modifiée en 2007.

Il est cependant certain que le constituant ne fait jamais de déclaration vaine. L'article 228, deuxième trait, lu avec l'article 48 montre la place que le constituant burundais attache

66 Commission Constitutionnelle, Rapport sur la démocratisation des institutions et de la vie politique au Burundi, Bujumbura, Août, 1991, p.100.

67 BADET, S.G., op.cit, p.168.

68 BADET, S.G., op.cit, p.189.

69 Idem, p. 170.

70 Codes et lois du Burundi (décembre 2006), p.18. 
aux droits fondamentaux et le rôle qu'il a entendu faire jouer à la Cour constitutionnelle pour les faire respecter. L'inexistence de règles procédurales de leur mise en œuvre ne constitue pas une difficulté insurmontable. Une juridiction constitutionnelle proactive pourrait elle-même y suppléer, par sa jurisprudence. La créativité est, en effet, de l'essence de la justice constitutionnelle. Le contrôle de constitutionnalité n'est-il pas lui-même une création du juge américain ${ }^{71}$ ? N'est-ce pas également le juge qui, en France, a étendu le contrôle de constitutionnalité aux libertés publiques ${ }^{72}$ ? N'est-ce pas aussi le juge qui, en Inde, a mis au point des règles procédurales nouvelles permettant la mise en œuvre effective des droits constitutionnels des classes sociales les plus marginalisées ${ }^{73}$, etc.?

Le droit comparé enseigne, en outre, que la protection juridictionnelle des libertés constitutionnelles ne se fait pas que par le contrôle des normes. Il en est ainsi notamment de l'amparo (littéralement recours en protection), très usité en Espagne et dans beaucoup d'Etats latino-américains. ${ }^{74}$ Il est ouvert à toute personne physique ou morale-de droit privé ou de droit public-qui s'estime lésée par un quelconque acte émanant des pouvoirs publics. L'acte attaqué en amparo peut être législatif, administratif ou même juridictionnel. ${ }^{75} \mathrm{La}$ seule condition est que le requérant prouve qu'il n'a pas pu obtenir satisfaction de la part du juge ordinaire. ${ }^{76}$ Avec le temps, l'amparo a fini par constituer, de loin, l'essentiel de l'activité du juge constitutionnel espagnol. Ainsi, par exemple, en 2009, sur les 10848 recours dont il a été saisi en 2009, 10 792, soit 99,48 \%, étaient des recours de ce type. ${ }^{77}$ Ceci a fait que, dans les termes de Pierre BON, le tribunal constitutionnel espagnol soit, avant tout, le garant du respect des droits fondamentaux par les actes non législatifs des pouvoirs publics et très accessoirement le garant du respect de l'intégralité des dispositions de la Constitution par les actes ayant force de loi. C'est évidemment une constatation importante pour qui considère que la raison d'être des juridictions constitutionnelles est de contrôler la constitutionnalité des lois. ${ }^{78}$

Il est légitime de croire que le constituant burundais de 2005 voulait également que la juridiction s'occupe essentiellement de faire respecter les libertés qu'il consacrait, sans que compte ne soit tenu de la nature juridique de l'acte qui y porterait atteinte. C'est sûrement

71 Cour Suprême des Etats-Unis : Marbury vs. Madison. Pour lire cette décision, allez notamment ici : http://www.lawnix.com.

72 Voir décision du Conseil constitutionnel n 71-44 DC du 16 juillet 1971, plus couramment désignée comme la décision «Liberté d'association». Pour l'integralité de la décision, allez ici : http:// www.conseil-constitutionnel.fr.

73 Banduha, supra n. 10, A.I.R., 815, S.C.C., 189. In Cassels, J., op.cit, p.501.

74 Pour une ample présentation du recours en amparo, voir : Tschentscher, A. et Lehner, C.: The Latin American Model of Constitutional Jurisdiction: Amparo and Judicial Review. Research Paper No. 2296004 ssrn.com (November, 20, 2014).

75 BON, P. La question d'inconstitutionnalité en Espagne, Pouvoirs-137, p. 123.

76 Ibidem.

77 Ibidem.

78 BON, P. La question d'inconstitutionnalité en Espagne, Pouvoirs-137, p. 124. 
pour cette raison que l'article 228, 2ème trait, de la constitution confère à la Cour le pouvoir de faire respecter la constitution-avec la charte des droits-non seulement par le législateur, mais aussi par "les organes de l'Etat et les autres institutions. " ${ }^{79}$ N'est-ce pas d'ailleurs là une des conséquences pratiques de la constitutionnalisation-par l'article 19-des droits de l'homme reconnus par les conventions internationales?

Il est cependant intéressant de relever que dans l'histoire de la justice constitutionnelle burundaise, la seule occasion à laquelle la compétence d' « assurer le respect » de la constitution a été mise en œuvre n'avait rien à voir avec la protection des libertés ou des droits fondamentaux. A la suite de la destitution de Monsieur Hussein Radjabu comme président du parti CNDD-FDD, une partie des parlementaires élus sur les listes de ce parti (22 députés), mécontents de cette décision, commencèrent à boycotter les activités de son groupe parlementaire. Les membres de ce groupe de 22 députés avaient, par ailleurs, soit été expulsés, soit démissionné du parti. Pour sortir de cette crise, le président du parti CNDD-FDD demanda, en date du 23 mai 2008, au Président de l'Assemblée Nationale de saisir la Cour constitutionnelle pour lui demander si les sièges de ces 22 députés étaient régulièrement occupés. Il le fit en date du 30 mai 2008. Six jours après, c'est-à-dire le 5 juin 2008, la Cour rendit son arrêt, en déclarant, comme on le lui avait demandé, que les sièges des 22 députés étaient irrégulièrement occupés. ${ }^{80}$ L'analyse détaillée de cet arrêt dépasse l'objet de cet article. Il est cependant intéressant d'observer la manière dont la Cour avait fondé sa compétence. Il s'agissait d'une question assez délicate et la Cour n'avait jamais eu à traiter de problèmes similaires. Elle fonda sa compétence sur l'article 228, deuxième trait de la constitution en vertu de laquelle la Cour est compétente pour « assurer le respect de la présente constitution, y compris la Charte des droits fondamentaux, par les organes de l'Etat et les autres institutions». Etrange tout de même que la Cour prit le député occupant son siège-et non l'A.N- comme un « organe » de l'Etat.

Un commentateur a montré combien cet arrêt aurait pu positivement influencer le contentieux constitutionnel des droits de l'homme. ${ }^{81}$ Le raisonnement suivi par la Cour dans cet arrêt lui permettrait, en effet, de contrôler tous les actes juridiques et même matériels des organes de l'Etat qui porteraient atteinte à la constitution, et notamment à la charte des droits. Le fait est cependant que, de la date de cet arrêt (5.6.2008) au moment où la version définitive de cette réflexion est déposée (25.1.2015), la Cour n'a rendu aucun autre arrêt en fondant sa compétence sur la même disposition.

Le juge constitutionnel burundais pourrait bien enlever toutes les restrictions à sa saisine et mettre entièrement en œuvre la compétence qu'il tire de l'article 228, 2ème trait,

79 Article 228, deuxième trait.

80 Cour constitutionnelle du Burundi; RCCB 213 du 5.6.2008.

81 Vandeginste, S., Pouvoir et droit au Burundi: un comment aire (principalement) juridique sur l'arrêt du 5 juin 2008 de la Cour Constitutionnelle dans l'affaire RCCB 213(www.uantwerpen.be): date de relecture : 20.11.2014. 
sans aucun risque d'être submergé de requêtes. Le risque de se retrouver unique régulateur $\mathrm{du} \ll \mathrm{jeu} »$ politique est également insignifiant-si pas inexistant-.

2. L'insignifiance du volume de travail de la Cour constitutionnelle et l'inexistence du risque de judiciarisation excessive du politique

La légalisation du politique et sa judiciarisation conséquente est un concept actuellement à la mode. ${ }^{82}$ Les systèmes politiques contemporains sont marqués par une montée en puissance frappante de la place accordée au juge au sein de l'ordre interne. Fatima, D. observe que cette forte ascension du juge conduit à une judiciarisation de plus en plus étendue de la vie sociale. ${ }^{83}$ Le juge dit et même crée du droit en ce qu'il est amené à codifier les valeurs de notre temps. Il exerce ainsi, ce que Robert Badinter appelle un «pontificat laïc.» ${ }^{84}$

A certaines occasions du conflit burundais, la judiciarisation du politique a été redoutée par une certaine élite politique. A titre purement exemplatif, l'ancien Président de la République Sylvestre Ntibantunganya admet que la suspension des cinq membres tutsi de la Cour constitutionnelle décidée en date du 29 janvier 1994-après la démission de deux autres membres-Hutu-était justifiée par la tendance de cette Cour à " gêner l'action du gouvernement engagé dans la normalisation de la vie politique nationale.» ${ }^{85}$

Le juge trop soucieux de la «paix » avec les deux autres pouvoirs peut ainsi chercher à s'autocensurer par une interprétation ultra-restrictive des règles de saisine. ${ }^{86}$ Assez logiquement cependant, la crainte de la judiciarisation du politique est irrationnelle. L'intervention du juge dans la vie sociopolitique d'un Etat est tout simplement inévitable. Elle est de l'essence du constitutionnalisme.

Tout compte fait cependant, la judiciarisation excessive du politique a tout l'air d'un débat simplement académique, en ce qui concerne le Burundi. La cour constitutionnelle aurait tort de prétendre y faire face en limitant, plus qu'il ne faut, sa saisine par les particuliers. La cour constitutionnelle du Burundi est parmi les juridictions les moins occupées du pays. Elle est rarement saisie et le gros des saisines concernent l'exercice des compétences autres que le contrôle normatif de constitutionnalité. Les saisines en contrôle de la constitutionnalité des lois viennent, le plus souvent, du Président de la République pour contrôle a priori des lois organiques. Nous l'illustrons par les cinq tableaux suivants. Nous suivons, pour cela, ce qu'il est fondé de considérer comme les moments les plus importants de la vie

82 Voir notamment Mandel, M.: The Rule of Law and the Legalization of Politics in Canada," 13 Int7 J. Sociol. L. 273 (1985).in CASSELS, J. op.cit, pp. 495-519.

83 Fatima, D.: Le juge constitutionnel et la construction de l'État de droit au Sénégal in Abbink, J. \& Bruijn, M., op.cit, p.259.

84 Badinter, R. \& Breyer, S. (éd.): Les entretiens de provence, le juge dans la société contemporaine, Fayard, Paris, 2003.

85 Ntibantunganya, S. Une démocratie pour tous les Burundais, La guerre ethno-civile s'installe. 1993-1996, volume 2, Paris, L'Harmattan, 1999, 15.

86 Voir notamment Cassels, J., op.cit, 502. 
de la Cour constitutionnelle burundaise. Ils montreront, entre autres, que l'enthousiasme pour la saisine de cette juridiction est allé diminuant.

a. La Cour « première génération »: du 13 mars 1992 au 13 septembre 1996

\begin{tabular}{|l|l|}
\hline OBJET DE LA REQUETE & $\begin{array}{l}\text { NOMBRE DE } \\
\text { REQUETES }\end{array}$ \\
\hline Contrôle de constitutionnalité : & 32 \\
-Sur saisine du Président de la République & 13 \\
-Sur saisine des particuliers (individus et personnes morales) & 19 \\
\hline Contrôle de constitutionnalité des règlements, y compris le contentieux électoral & 9 \\
\hline Autres décisions liées aux élections & 8 \\
\hline Interprétation de la constitution & 4 \\
\hline Interprétation des arrêts de la cour constitutionnelle & 1 \\
\hline Autres matières & 2 \\
\hline TOTAL & 56 \\
\hline
\end{tabular}

87

En trois ans et demi, la Cour a rendu 56 arrêts dont plus de la moitié (32) en matière de contrôle de constitutionnalité des lois. Dans ces 32 saisines en inconstitutionnalité, 19 provenaient des particuliers.

Le tableau montre qu'aux tout premiers jours de la vie de la Cour, les plaideurs avaient saisi son potentiel d'être une véritable « cour des droits de l'homme.» Le taux de sa saisine par les particuliers était aussi, compte tenu de sa nouveauté, assez remarquable. ${ }^{88}$ Les choses ne tardèrent pas d'évoluer en sens inverse.

87 S.Vandeginste, op.cit, p. 510.

88 S.Vandeginste, op.cit, p. 510 
b. La Cour « deuxième génération »: du 6 juin 1998 au 28 octobre 2001

\begin{tabular}{|c|c|}
\hline OBJET DE LA REQUETE & $\begin{array}{l}\text { NOMBRE DE } \\
\text { REQUETES }\end{array}$ \\
\hline $\begin{array}{l}\text { Contrôle de constitutionnalité : } \\
\text {-Sur saisine du Président de la République } \\
\text {-Sur saisine du Président de l'Assemblée Nationale de Transition(ANT) } \\
\text {-Sur saisine des particuliers (individus et personnes morales }\end{array}$ & $\begin{array}{l}3 \\
1 \\
0\end{array}$ \\
\hline Contrôle de constitutionnalité des règlements & 1 \\
\hline Constitutionnalité du règlement intérieur de l'ANT & 2 \\
\hline $\begin{array}{l}\text { Conformité de la désignation/remplacement de membres de l'ANT à l'Acte } \\
\text { constitutionnel de Transition/déclaration de vacance de siège à l'ANT }\end{array}$ & 15 \\
\hline Interprétation de l'Acte constitutionnel de Transition & 1 \\
\hline TOTAL & 23 \\
\hline
\end{tabular}

La Cour constitutionnelle « deuxième génération $»^{90}$ rendit, en trois ans, 23 arrêts. Elle ne fut saisie en contrôle de constitutionnalité des lois et règlements qu'à 4 reprises, dont une seule fois par un particulier.

Il transparait, en comparant les deux tableaux précédents, qu'à partir de 1998, la Cour avait cessé d'être une «cour des droits de l'homme ». Sur une période de plus de 3 ans, elle n'a été saisie qu'une fois par un particulier. Ce recul de l'enthousiasme dans la saisine de cette juridiction s'explique aussi bien juridiquement que politiquement. D'un point de vue juridique, la jurisprudence de la Cour avait amené les praticiens du Droit à douter des chances de faire aboutir leurs demandes. D'un point de vue politique, le putch du 25 juillet 1996 avait mis un frein à l'élan de libéralisme politique déclenché en 1993. En outre, l'Assemblée Nationale-majoritairement composée de députés issus du FRODEBU-avait signé, avec l'Exécutif putschiste du 25 juillet 1996, un accord de «partenariat politique de transition » en date du 8.6.1998. Ce dernier texte faisait qu'il n'y ait pratiquement plus d'opposition parlementaire. L'on peut aussi oser l'observation que la séparation des pouvoirs législatif et exécutif avait été sérieusement mise à mal. Les remises en cause juridictionnelles des actes d'une branche du pouvoir par une autre étaient alors difficilement envisageables. La Cour ne fut même pas vue comme un levier politique utile par ceux qui rejetaient cet accord. ${ }^{91}$

89 Idem, p.515.

90 L'expression « génération » n'a rien à voir avec le changement de juges membres de la C.C. Elle fait plutôt allusion aux textes fondamentaux dont la C.C avait à assurer le respect.

91 Pour les détails, voir Vandeginste, S. Law as a source and instrument of transitional justice in Burundi, Thèse de doctorat en droit, Université d'Anvers, 2008, Anvers, 2009, p. 516(inédit). 
c. La Cour « troisième génération »: du 28 octobre 2001 au 18 mars 2005

\begin{tabular}{|c|c|}
\hline OBJET DE LA REQUETE & $\begin{array}{l}\text { NOMBRE DE } \\
\text { REQUETES }\end{array}$ \\
\hline $\begin{array}{l}\text { Contrôle de constitutionnalité : } \\
\text {-Sur saisine du Président de la République : - } \\
\text {-Sur saisine des particuliers (individus et personnes morales) }\end{array}$ & $\begin{array}{l}20 \\
16 \\
4\end{array}$ \\
\hline Contrôle de constitutionnalité des règlements & 0 \\
\hline $\begin{array}{l}\text { Contrôle de la constitutionnalité des règlements intérieurs de l'Assemblée Natio- } \\
\text { nale de Transition(ANT) et du Senat de Transition(ST) }\end{array}$ & 4 \\
\hline $\begin{array}{l}\text { Conformité de la désignation/remplacement de membres de l'ANT/ST à la } \\
\text { constitution de transition/déclaration de vacance de siège à l'ANT/ST }\end{array}$ & 56 \\
\hline $\begin{array}{l}\text { Conformité de la constitution post-transition à l'Accord d'Arusha pour la paix et } \\
\text { la réconciliation }\end{array}$ & 5 \\
\hline Interprétation de la constitution de transition & 5 \\
\hline Interprétation des arrêts de la Cour constitutionnelle & 3 \\
\hline Proclamation des résultats de référendum & 4 \\
\hline Autres matières & 4 \\
\hline Information indisponible & 2 \\
\hline TOTAL & 100. \\
\hline
\end{tabular}

92

En trois ans et demi, la Cour ne fit pas sensiblement mieux qu'aux périodes précédentes. Sur 100 affaires, 20 seulement concernaient le contrôle normatif de constitutionnalité. Sur ces 20 saisines, 4 seulement venaient des particuliers.

Pour les mêmes raisons que précédemment, la Cour « troisième génération » n'avait pas suscité beaucoup d'enthousiasme. Avec la constitution du 18 mars 2005, les choses ne s'améliorent pas, non plus.

d. La Cour « quatrième génération » : du 18 mars 2005 au 1er Juin 2008

\begin{tabular}{|l|l|}
\hline Objet de la requête & $\begin{array}{l}\text { Nombre de } \\
\text { requêtes }\end{array}$ \\
\hline Contrôle de la constitutionnalité des lois : & 17 \\
-Sur saisine du Président de la République & 13 \\
-Sur saisine d'un quart des députés & 1 \\
-Sur saisine des individus/personnes morales & 3 \\
\hline Contrôle de la constitutionnalité des règlements & 4 \\
\hline Contrôle de la constitutionnalité des règlements intérieurs de l'A.N et du Sénat & 5 \\
\hline
\end{tabular}




\begin{tabular}{|l|l|}
\hline Objet de la requête & $\begin{array}{l}\text { Nombre de } \\
\text { requêtes }\end{array}$ \\
\hline $\begin{array}{l}\text { Contrôle de la régularité des élections, contentieux électoral et proclamation des } \\
\text { résultats des élections et de referenda }\end{array}$ & 25 \\
\hline Déclaration de vacance de siège à l'Assemblée Nationale et au Sénat & 27 \\
\hline Interprétation de la constitution & 5 \\
\hline Interprétation des arrêts de la cour constitutionnelle & 0 \\
\hline Autres & 3 \\
\hline Information non disponible & 3 \\
\hline Total & 89 \\
\hline
\end{tabular}

93

En trois ans et demi, la Cour rendit 89 arrêts dont 21 en contrôle de constitutionnalité. Les saisines par les particuliers furent cependant limitées à 5 .

e. La Cour « cinquième génération »: du 2 juin 2008 au 31 décembre 2014

\begin{tabular}{|l|l|}
\hline Objet de la requête & $\begin{array}{l}\text { Nombre de } \\
\text { total }\end{array}$ \\
\hline $\begin{array}{l}\text { Contrôle de la constitutionnalité des lois : } \\
\text {-Sur saisine du Président de la République }\end{array}$ & 44 \\
-Sur saisine d'un quart des députés & 19 \\
-Sur saisine des individus/personnes morales & 0 \\
\hline $\begin{array}{l}\text { Autres requêtes (constat d'occupation irrégulière de siège, interprétation de la } \\
\text { constitution, constat de vacance de siège de députés ou sénateurs, contentieux } \\
\text { électoral) }\end{array}$ & 25 \\
\hline Total & 41 \\
\hline
\end{tabular}

En 6 ans et demi, la Cour constitutionnelle a rendu 85 arrêts, dont 44 en contrôle de constitutionnalité. Sur les 44 saisines en contrôle de constitutionnalité, 25 provenaient des particuliers.

L'on peut avoir l'illusion que la Cour constitutionnelle a été beaucoup sollicitée pendant cette période et que son volume d'affaires en rapport avec la constitutionnalité des lois s'est relativement accru. La vérité est que le changement est plutôt insignifiant. En effet, en ce qui concerne le volume général des affaires, il faut remarquer que la Cour n'est généralement occupée que pendant les années d'élections et par le contentieux électoral. Il suffit,

93 S.Vandeginste, op.cit, p.520.

94 Registre des requêtes adressées à la Cour constitutionnelle : consulté par l'auteur de cet article en date du 2.01.2014. 
pour s'en convaincre, de comparer les années 2010 et 2011. En 2010, année électorale, la Cour a reçu 24 requêtes. Au cours de l'année immédiatement suivante(2011), elle n'a été saisie qu'à 6 reprises. La proportion d'affaires relatives au contrôle de constitutionnalité ne doit pas, non plus, impressionner outre mesure. Comme en d'autres périodes, en effet, les saisines du Président de la République n'ont concerné que les lois organiques pour lesquelles le contrôle de constitutionnalité est obligatoire. Le nombre d'affaires connues par la Cour sur saisine des particuliers n'est pas significatif : 25 affaires en six ans (4 affaires/an) constituent un chiffre pas du tout impressionnant. En outre, il ne traduit, même pas, le nombre de textes normatifs attaqués en inconstitutionnalité. En effet, la Cour numérote les requêtes- peut-être assez logiquement-en fonction de l'identité du requérant et non de l'objet de la requête. Il en résulte qu'elle peut enregistrer des requêtes sous des numeros différents alors qu'elles portent le meme objet. Il en a été ainsi notamment des requêtes contre l'A.R du 21 novembre 1925 relatif à la procédure de vente par voie parée et spécialement les articles 4, 10 et 23 de cet arrêté 95 .

L'observation de ces données chiffrées montrent que le risque que le plaideur burundais n'abuse de l'ouverture du prétoire constitutionnel est quasi-nul. Le Burundais n'est pas procédurier et le paysage politico-associatif burundais ne connait pas de «busybodies ». Il n'en va pas de même dans d'autres pays africains. Au Bénin, par exemple, BADET rapporte qu'entre juin 1993, date d'entrée en fonction de la Cour constitutionnelle définitive, et décembre 2008, la Cour a rendu 2400 décisions dont 1728 en contrôle de constitutionnalité. ${ }^{96}$ Dans ce même pays, en fin 2005, sur 1218 décisions rendues par la Cour constitutionnelle, 1012 avaient eu pour origine des requêtes de citoyens, soit plus de $83 \% .{ }^{97}$

La situation devient beaucoup plus préoccupante pour le Burundi lorsque l'on sait que les institutions et mandataires habilités à saisir la juridiction constitutionnelle-autres que les individus et les personnes morales-ne la saisissent presque jamais, sauf lorsqu'elles y sont constitutionnellement obligées.

Les tableaux ci-dessus auront permis de constater que le Président de la République n'a jamais saisi la Cour en contrôle de constitutionnalité que pour la vérification de constitutionnalité des lois organiques. Il y est, en effet, on l'a déjà souligné, constitutionnellement obligé par l'article 228, al.2.

Les députés et/ou sénateurs de l'opposition, ne recourent presque jamais à la Cour. Alors que dans certains autres pays, les députés de l'opposition usent de la possibilité de saisir la juridiction constitutionnelle pour prolonger et éventuellement gagner, au prétoire, le débat perdu à l'hémicycle ${ }^{98}$, les parlementaires burundais n'ont pas cette culture. De 1992 à décembre 2014, ils n'ont saisi la Cour qu'à deux reprises. La première fois, c'était

95 Cour constitutionnelle du Burundi; RCCB 277du 17 février 2014(inédit).

97 Ibidem.

98 D'après JAN, P. (op.cit, p.4), les saisines parlementaires constituent, en France, plus de 93,3\%- de l'activité du conseil constitutionnel. 
pour remettre en cause la constitutionnalité de la loi créant la cour et la brigade anti-corruption. ${ }^{99}$ La deuxième fois, il s'agissait de contester le décret nommant Madame Marine BARAMPAMA au poste de deuxième vice-président de la République. ${ }^{100} \mathrm{Il}$ faut cependant reconnaitre également qu'au Burundi, il n'est pas courant que l'opposition atteigne le nombre utile de sièges-un quart des membres de la chambre-.

L'ombudsman et le Ministère Public, n'ont, en ce qui les concerne, jamais saisi la Cour constitutionnelle.

Il est donc évident que le constitutionnalisme ne pourra être vivant et l'Etat de droit devenir une réalité au Burundi que par l'action juridictionnelle des particuliers.

\section{CONCLUSION}

Cet article aura mis en évidence que la constitution burundaise du 18.5.2005 a voulu grandement ouvrir les portes de la justice constitutionnelle aux particuliers. Elle prolongeait, par ailleurs, une tendance déjà très prononcée dans la constitution du 13.3.1992. Le constituant donnait ainsi à la nation l'un des ingrédients les plus importants pour l'instauration et l'affermissement d'un état de droit.

La réflexion aura cependant montré que, par son interprétation excessivement restrictive de la notion d'intérêt à agir et par sa détermination hyper-étroite de la norme contrôlable sur saisine du particulier, la cour constitutionnelle a réduit cet énorme potentiel à très peu de choses.

La réflexion aura également mis en évidence que cette jurisprudence est incompatible avec l'esprit de la constitution et avec le constitutionnalisme moderne. Pire, elle n'est même pas politiquement justifiée par ce qu'elle est censée répondre à un risque inexistant dans le contexte burundais : l'abus de ce recours par le justiciable.

Un changement de cap jurisprudentiel est alors indispensable pour faire bénéficier aux Burundais et habitants du Burundi l'immense trésor de libertés publiques et de droits sociaux dont regorge la loi fondamentale. L'article aura indiqué, du point de vue de l'auteur, les rectifications qui s'imposent.

Tout ne dépendra cependant pas du juge. En effet, celui-ci n'agit que sur saisine. Au Burundi, l'un des obstacles sociologiques majeurs à la saisine du juge est l'ignorance. Le manque de confiance dans les institutions étatiques-en ce compris les cours et tribunaux-est aussi un facteur déterminant. Cet article n'a cependant pas discuté de cette problématique. Nous invitons très vivement d'autres chercheurs intéressés par la problématique de l'état de droit au Burundi à s'y pencher.

99 Cour constitutionnelle du Burundi: RCCB 178 du 17 octobre 20061105.

100 Vandeginste, S., op.cit, p.522. 


\section{INDICATIONS BIBLIOGRAPHIQUES}

I. Textes normatifs

A. Textes constitutionnels

1. Ordonnance législative $\mathrm{n}^{\circ} 01 / 20$ du 30 janvier 1962 portant constitution du royaume du Burundi, in B.O.R.U, 1962, p.67 et B.O.B., 1962, p.12.

2. Constitution burundaise du 16.10.1962, B.O.B nº 1 bis/63, pp.1-13.

3. Constitution burundaise du 11.7.1974, in B.O.B. $\mathrm{n}^{\circ}$ 8/74, pp.211-216.

4. Décret-loi $\mathrm{n}^{\circ} 1 / 23$ du 20 novembre 1981 portant promulgation de la Constitution burundaise du 1.11.1981, in B.O.B. n ${ }^{\circ} 9$ à 12/1981, pp.407-423.

5. Décret-loi $n^{\circ} 1 / 06$ du 13 mars 1992 portant promulgation de la constitution de la République du Burundi. B.O.B n 4/92, pp.97-155.

6. Décret-loi $\mathrm{n}^{\circ} 1 / 001 / 96 \mathrm{du} 13$ septembre 1996 portant organisation du système institutionnel de transition, B.O.B. $n^{\circ} 11 / 1996$, pp.553-565.

7. Décret-loi $\mathrm{n}^{\circ} 1 / 008$ du 6.6.1998 portant promulgation de l'Acte constitutionnel de transition de la République du Burundi, B.O.B. n 7/98, pp.468-483.

8. Loi $\mathrm{n}^{\circ} 1 / 017$ du 28 octobre 2001 portant promulgation de la constitution de transition de la République du Burundi, B.O.B. n 10/2001, pp.1269-1302.

9. Loi $\mathrm{n}^{\circ} 1 / 024$ portant du 21 novembre 2003 portant amendement à la constitution de la République du Burundi. B.O.B n ${ }^{\circ} 11$ bis/2003, pp.781-783.

10. Loi ${ }^{\circ} 1 / 010$ du 18 mars 2005 portant promulgation de la constitution de la République du Burundi (Codes et lois du Burundi (31 décembre 2006, Tome I, pp.1-23.).

B. Textes législatifs et réglementaires

1. Loi $\mathrm{n}^{\circ} 1 / 07$ du 25 février 2005 régissant la Cour Suprême Codes et lois du Burundi (31 décembre 2006, Tome II, pp.170-182)

2. Loi $\mathrm{n}^{\circ} 1 / 08 \mathrm{du} 17$ mars 2005portant Code de l'organisation et de la compétence judiciaires (Codes et lois du Burundi (31 décembre 2006, Tome II, pp.159-170).

3. Loi $\mathrm{n}^{\circ} 1 / 03$ du 11 janvier 2007 portant modification de certaines dispositions de la loi $\mathrm{n}$ ${ }^{\circ} 1 / 018$ du 19 décembre 2002 portant Organisation et Fonctionnement de la Cour Constitutionnelle ainsi que là procédure applicable devant elle (pas publiée)

4. Arrêté Royal n 100/34 du 23.11. 1966 portant érection du parti « Unité et Progrès National » en parti unique national, in B.O.B. $\mathrm{n}^{\circ}$ 9/66, p.315.

5. Décret $\mathrm{n}^{\circ} 100 / 02$ du 03 janvier 2006 portant immunité provisoire des prisonniers politiques détenus dans les maisons de détention de la République du Burundi B.O.B n ${ }^{\circ} 1 / 2006$, p. 2 . 
II. Jurisprudence

Cour constitutionnelle du Burundi : RCCB 1 du 13.4.1992, inédit. Cour constitutionnelle du Burundi : RCCB2 du 14. 4.1992, inédit. Cour constitutionnelle du Burundi : RCCB 3 du 19.10.1992, B.O.B. n 2/93, pp. 55-56. Cour constitutionnelle du Burundi : RCCB 7 du 7.12.1992. inédit Cour constitutionnelle du Burundi : RCCB 8 du 30.3.1993, inédit. Cour constitutionnelle du Burundi : RCCB 27 du 2.08.1993, inédit. Cour constitutionnelle du Burundi : RCCB 28 du 10.8.1993, in B.O.B. n 4/94, pp.5-9. Cour constitutionnelle du Burundi : RCCB 39 du 18.04. 1994, B.O.B n 6/94, pp.3-5. Cour constitutionnelle du Burundi : RCCB 54 du 5.10.1995 in B.O.B. 12/95, pp. 427-432.

Cour constitutionnelle du Burundi : RCCB 58 du 21.7.2003, inédit. Cour constitutionnelle du Burundi : RCCB126 du 11.7.2005, inédit Cour constitutionnelle du Burundi : RCCB 160-161 du 2.3.2006, in B.O.B n ${ }^{\circ} 6 \mathrm{bis} / 2012$, pp.1031-1041.

Cour constitutionnelle du Burundi : RCCB 174 du 22/8/2006, in B.O.B nºbis/2012, pp. 1053-1054.

Cour constitutionnelle du Burundi : RCCB 178 du 17.10.2006 in B.O.B., pp. 1057-1059.

Cour constitutionnelle du Burundi : RCCB 213 du 5.6.2008, inédit, Cour constitutionnelle du Burundi: RCCB 228 du 19.4.2010 in B.O.B 11 bis/2012, p. 1848

Cour constitutionnelle du Burundi : RCCB 256 du 9.3.2012., in B.O.B n5/2013, pp. 749-752.

Cour constitutionnelle du Burundi : RCCB 269, inédit.

Cour constitutionnelle du Burundi RCCB 271 du 8 novembre 2013, inédit.

Cour constitutionnelle du Burundi : RCCB 277 du 17 février 2014, inédit.

\section{Doctrine}

A. Ouvrages

1. Abbink, J. \& Bruijn, M.(ed.) : Land, Law and Politics in Africa, BRILL, Leyde, 2011.

2. Badinter, R. \& Breyer, S.(éd.): Les entretiens de provence, le juge dans la société contemporaine, Fayard, Paris, 2003.

3. Blondel, J. (ed.): Comparative government. Mac Millan and co, Ltd, London.1969.

4. Conac, G. Les cours suprêmes en Afrique, Economica, Paris, 1987.

5. Dehousse, F : Introduction au droit public, Editions Collection Scientifique de la Faculté de Liège, 1995, 472p.

6. Grosser, A. Au nom de quoi? Fondement d'une morale politique. Editions du Seuil, Paris, 1969, 311p. 
7. Guchet, Y. Eléments de droit constitutionnel, Edition Albatros, Paris, 1981, 587p.

8. Hauriou, A. Droit constitutionnel et Institutions politiques, 4e éd, Montchrestien, Paris, 1970.

9. Lerat, C. La Cour suprême des Etats-Unis : Pouvoirs et évolution historique, 4eme édition, 33405, Talence cedex, P.U.F, Bordeaux, 1990, 327p.

10. Ntibantunganya, S. Une démocratie pour tous les Burundais, La guerre ethno-civile s'installe. 1993-1996, volume 2, Paris, L'Harmattan, 1999.

11. Viljoen, F.: International Human Rights Law in Africa: Oxford University Press.

B. Articles et contributions (chapitres) aux ouvrages collectifs

1. Bahdon, A. : La juridiction constitutionnelle en République de Djibouti : Enjeux et perspectives de la garantie du respect des droits et des principes fondamentaux : Questiones constitucionales, Num 15, julio-diciembre 2006.

2. Barry, H. A re-examination of the case for locus standi in public law, Cambrian Law review, 1997, pp. 83-104.

3. Bon, P. La question d'inconstitutionnalité en Espagne, Pouvoirs-137, 2011, pp. 123-141.

4. Brunet, P. Le juge constitutionnel est-il un juge comme les autres? Réflexions methodologiques sur la justice constitutionnelle in Grewe, C., Jouanjan, O., Maulin, E. \& Wachsmann, P. (éd.) : La notion de justice constitutionnelle, Dalloz, Paris, 2005, pp. 115-135.

5. Cassels, J.: Judicial Activism and Public Interest Litigation in India: Attempting the impossible? The American journal of comparative Law, vol. 37, pp. 495-519.

6. Christensen, C. \& Odinkalu, C.A. : The African Commission on Human and Peoples' Rights, Human Rights Quarterly, vol.20, pp.235-280.

7. Fatima, D. : Le juge constitutionnel et la construction de l'État de droit au Sénégal 1 in Abbink, J. \& Bruijn, M. Law, land and politics in Africa, pp.258-286.

8. Harperin, $J . \mathrm{L}$ : La question prioritaire de constitutionnalité : une révolution dans l'histoire du droit français? in Les Cahiers du Conseil constitutionnel, 2010, pp. 31-34.

9. HOLO, T. Emergence de la justice constitutionnelle, Pouvoirs, 2009/2, N¹29, pp. 101-102.

10. Kanté, B. : La production d'un nouveau constitutionnalisme en Afrique : Internationalisation et régionalisation du droit constitutionnel in Abbink, J. \& Bruijn M.(éd.) Land, Law and Politics in Africa, pp. 240-257.

11. LOEWENSTEIN, K. The « living » constitution: Shadow and substance, in Blondel,J. (ed.): Comparative government. Mac Millan and co, Ltd, London.1969.

12. Mandel, M.: The Rule of Law and the Legalization of Politics in Canada," 13 Int7 J. Sociol. L. 273 (1985) inCASSELS, J. op.cit, pp. 495-519). 
13. SOMA, A. : Modélisation d'un système de justice constitutionnelle pour une meilleure protection des droits de l'homme : trans-constitutionnalisme et droit constitutionnel comparé, Revue trimestrielle des droits de l'homme, 2009, vol. 20, no. 78, p. 437-466.

14. Tokar, J.J: Administrative Law: Locus standi in judicial review proceedings, in Manitoba Law Journal, Vol. 14, pp.209-.243.

15. Vandeginste, S., Pouvoir et droit au Burundi: un comment aire (principalement) juridique sur l'arrêt du 5 juin 2008 de la Cour Constitutionnelle dans l'affaire RCCB 213(www.uantwerpen.be): date de relecture : 20.11.2014.

A. Thèses de doctorat et mémoires non publiés

1. BADET, S.G. : Contrôle intra normatif et contrôle ultra normatif de constitutionnalité: Contribution à l'identification des sous catégories du modèle kelsénien de justice constitutionnelle à partir des systèmes belge et béninois. Thèse de doctorat, Université Catholique de Louvain, A.A. : 2011-2012.

2. JAN, P. : L'accès au juge constitutionnel : modalités et procédures, Rapport du deuxième congrès de l'ACCPUF (inédit).

3. Ntahiraja, B. Le contrôle de la constitutionnalité des lois en droit comparé. Mémoire, Université du Burundi, Faculté de Droit, 2007, inédit.

4. Vandeginste, S. Law as a source and instrument of transitional justice in Burundi, Thèse de doctorat en droit, Université d'Anvers, 2009, inédit.

D. Manuels, Rapports, articles de presse, exposés de séminaires et autres documents

1. Commission Constitutionnelle, Rapport sur la démocratisation des institutions et de la vie politique au Burundi, Bujumbura, Août, 1991.

2. Gallet, J.L : L'accès à la justice dans les traditions constitutionnelles nationales - La qualité pour agir dans l'ordre juridique français (document du séminaire de l'ERA portant sur « L'accès à la justice dans le droit communautaire : le rôle du juge national », organisé en coopération avec la Cour de cassation les 1er et 2 juin 2006 à Paris.).

3. JAN, M.P : L'accès au juge constitutionnel : modalités et procédures (rapport du deuxième Congrès de l'A.C.C.P.U.F.).

4. Kenneth, R. Thomas, Selected theories of constitutional interpretation, Congressional Research Services (CRS Report for US Congress)

5. KU J. What Does China Mean When It Celebrates the "International Rule of Law"? www.opinio juris opinion juris.org (30/10/2014).

6. Queensland Public Interest Law Clearing House Incorporated: Standing in public interest cases, July, 2005, 
7. Sénat français : Les recours devant le juge constitutionnel, Documents de travail du Sénat, Série Législation Comparée ( $\mathrm{LC} \mathrm{n}^{\circ}$.208), septembre 2010.

8. Tafur Isabella: No more than a meddlesome busybody? Worshop paper: www.solicitorsjournal.com(august, 13, 2013). 


\section{Impressum}

\section{KAS African Law Study Library - Librairie Africaine d'Etudes Juridiques}

Herausgeber: Konrad-Adenauer-Stiftung e.V., Klingelhöferstraße 23, 10785 Berlin

Schriftleitung: RA Prof. Dr. Hartmut Hamann, Falkertstraße 82, D-70193 Stuttgart Telefon: +49 (o) 711 120950-30 | Fax : +49 (o) 711 120950-50

Mail: hamann@hamann-legal.de

Erscheinungsweise: 4 Ausgaben pro Jahr

Druck und Verlag: Nomos Verlagsgesellschaft mbH \& Co. KG - Waldseestr. 3-5 D-76530 Baden-Baden. Telefon +49-7221-2104-0 / Fax 49-7221-2104-27

E-Mail:nomos@nomos.de

Anzeigen: sales friendly Verlagsdienstleistungen - Inh. Frau Bettina Roos - Pfaffenweg 15 - D-53227 Bonn Telefon +49-228-97898-o / Fax +49-228-97898-20

E-Mail: roos@sales-friendly.de

Urheber- und Verlagsrechte: Die Zeitschrift und alle in ihr enthaltenen einzelnen Beiträge sind urheberrechtlich geschützt. Jede Verwertung außerhalb der engen Grenzen des Urheberrechtsgesetzes ist ohne Zustimmung des Verlages unzulässig. Das gilt insbesondere für Vervielfältigungen, Übersetzungen, Mikroverfilmungen und die Einspeicherung und Verarbeitung in elektronischen Systemen. Der Nomos Verlag beachtet die Regeln des Börsenvereins des Deutschen Buchhandels e.V. zur Verwendung von Buchrezensionen.

ISSN 2363-6262

\section{Nomos}

